\title{
Swelling and Degradation Characteristics of Crumb Rubber Modified Asphalt during Processing
}

\author{
Caiyun Xia, ${ }^{1}$ Mingyuan Chen $\mathbb{D D}^{2},{ }^{2}$ Jiuguang Geng, ${ }^{2}$ Xiaofeng Liao, ${ }^{1}$ and Zhongda Chen ${ }^{1}$ \\ ${ }^{1}$ School of Highway, Chang'an University, Xi'an, China \\ ${ }^{2}$ School of Materials Science and Engineering, Chang'an University, Xi'an, China \\ Correspondence should be addressed to Mingyuan Chen; chenmy@chd.edu.cn
}

Received 29 October 2020; Accepted 22 January 2021; Published 4 February 2021

Academic Editor: Guanyuan Zhao

Copyright (c) 2021 Caiyun Xia et al. This is an open access article distributed under the Creative Commons Attribution License, which permits unrestricted use, distribution, and reproduction in any medium, provided the original work is properly cited.

The existence of cross-linked structure in crumb rubber modified asphalt (CRMA) leads to the complexity of its system. According to the preparation test of CRMA under different processing technology, the influence of processing temperature and time on the properties and cross-linking structure of CRMA was analyzed. By analyzing the volume expansion rate of the rubber powder after swelling and the toluene insoluble content attenuation rate, the formation conditions of the macro-level cross-linked structure were determined, and the relationship model between the cross-linked structure and the rubber asphalt performance was established. The results show that the rubber particles can form a continuous phase structure after swelling. At this time, the expansion ratio of the swollen rubber powder was $1.76-2.14$. Too high temperature (above $200^{\circ} \mathrm{C}$ ) and too long time (above $60 \mathrm{~min}$ ) caused serious degradation of rubber. The suitable processing technology was stirring at $180-190^{\circ} \mathrm{C}$ for $45-60 \mathrm{~min}$.

\section{Introduction}

Crumb rubber modified asphalt (CRMA) is highly concerned by the road industry for its good temperature resistance, fatigue and skid resistance, reduction of road noise and road construction costs, and environmental advantages of waste recycling $[1,2]$. Waste tire rubber powder (vulcanized rubber) contains natural rubber, synthetic rubber, sulfur, carbon black, anti-aging agents, and other ingredients. These ingredients help to improve the quality of CRMA, including increasing the softening point, reducing penetration, increasing ductility, and improving the elastic recovery of CRMA [3]. However, crumb rubber (CR) is an inert polymer material. In the production process of modified asphalt, it needs strong stirring or mechanical shearing to be dispersed in the asphalt at high temperature. The current preparation process conditions are mainly determined with reference to engineering experience, and there is still a lack of theoretical basis for the interaction between rubber powder and asphalt. Many scholars have carried out research on the performance and engineering application of CRMA. A large number of research results have been obtained in the process conditions such as the preparation temperature and time of CRMA, as well as particle size and amount of rubber powder [4-8]. The reaction process of CRMA was closely related to the asphalt medium, reaction temperature, and reaction time, and the deeply degraded rubber powder can be quickly dispersed in the asphalt $[9,10]$.

The swelling and degradation behavior of CR in the asphalt greatly affects the modification effect and road performance of CRMA. In order to obtain high-performance CRMA, researchers have gradually begun to study the physicalchemical behavior and microscopic mechanism of CR and asphalt. Abdelrahman et al. [11] pointed out that there were rubber powder swelling and degradation behavior in CRMA. Frantzis et al. [12] and Cong et al. [13] used scanning electron microscope (SEM) to describe the swelling of rubber powder in hot asphalt. At the high temperature, the rubber powder absorbed the light components of the asphalt and expanded. The rubber powder particles contacted each other and were connected by the gel film to form a semi-solid continuous structure, which restricted the movement of the free asphalt and formed a continuous interlocking phase [14]. Cui et al. [6] 
and Dong et al. [15] used infrared spectroscopy (IR), thermogravimetry, and x-ray photoelectron spectroscopy (XPS) to analyze the impact of the microstructure of CR powder on the properties of CRMA and found that the swelling of rubber in the asphalt started from a rapid growth process and then tended to balance. Excessive temperature or time caused excessive degradation and agglomeration of rubber powder, or asphalt aging behavior, which would change the physical and chemical state of CRMA, resulting in performance degradation [16]. Besides, Wang et al. [17] and Ji et al. [18] used finite element model and Crank diffusion model to predict the expansion behavior of rubber in asphalt and proposed two swelling parameters-swelling coefficient and final swelling amount. However, the swelling and degradation characteristics of rubber powder in asphalt still lack rapid and intuitive analysis and evaluation. The physical and chemical properties and modification behavior of CRMA still need to be further studied.

In the paper, the changes in the cross-linking structure of CRMA during processing were evaluated quickly and intuitively from a macro perspective. CRMA was prepared by different processing temperature and time. Through penetration, softening point, ductility, viscosity, and resilience, the physical properties of CRMA under different processing temperature and time conditions were studied. In addition, the swelling and degradation of CRMA during processing were evaluated by the volume and mass of toluene insoluble matter. Besides, the correlation analysis of the applicability of the cross-linked structure test methods was carried out to determine the appropriate processing temperature and time from the perspective of rubber swelling and degradation in the CRMA. Finally, SEM was used for intuitive verification.

\section{Materials and Methods}

2.1. Raw Materials. The performance of base asphalt will directly affect the performance of crumb rubber modified asphalt (CRMA). The main properties of Shell-70 are shown in Table 1. The 40-mesh crumb rubber (CR) was produced in Sichuan Province, China. The basic physical properties of CR are shown in Table 2.

2.2. Preparation of CRMA. Prior to mixing, crumb rubber was preserved in the oven at $60^{\circ} \mathrm{C}$ for $30 \mathrm{~min}$ to remove residual water. Then, the dried crumb rubber ( $16 \%$ by the weight of base asphalt) was added to the heated asphalt. This blend was mixed at designated mixing temperature $\left(160^{\circ} \mathrm{C}\right.$, $180^{\circ} \mathrm{C}, 200^{\circ} \mathrm{C}, 220^{\circ} \mathrm{C}$, and $\left.240^{\circ} \mathrm{C}\right)$ and mixing time $(30 \mathrm{~min}$, $45 \mathrm{~min}, 60 \mathrm{~min}$, and $75 \mathrm{~min}$ ) using a high-speed shear mixer at a constant shearing speed of $5000 \mathrm{r} / \mathrm{min}$. Finally, the CRMA was stirred manually for $5 \mathrm{~min}$ to exhaust air.

2.3. Research Approach. The purpose of this study was to analyze the influence of different processing temperatures and times on the performance of CRMA with polynomial correlation. At the same time, the process of swelling and degradation of CRMA was judged from the macroscopic and microscopic perspectives. The material preparation and testing performed in this study are shown in Figure 1. Details are introduced in the following subsections.

2.3.1. Physical Properties. The physical properties of asphalt, including penetration at $25^{\circ} \mathrm{C}$, softening point, ductility at $5^{\circ} \mathrm{C}$, viscosity at $180^{\circ} \mathrm{C}$, and resilience at $25^{\circ} \mathrm{C}$, were tested in accordance with ASTM D5, ASTM D36, ASTM D113, ASTM D2196, and ASTM D5329, respectively.

\subsubsection{Degradation and Swelling of Crumb Rubber.} Crumb rubber would continue to swell and degrade during processing. Vulcanized rubber powder is basically insoluble in toluene, and the soluble part increases after desulfurization (S-S/C-S). The swelling and degradation of rubber can be evaluated by quantitatively analyzing the volume and mass loss of toluene insoluble matter (TIM) $[19,20]$. During CRMA processing, after reaching the specified time each time, without affecting the stirring, the CRMA with a certain mass $\left(M_{0}\right)$ was directly weighed out twice: Part a and Part b. Among them, Part a and Part b were used for swelling test and degradation test of crumb rubber, respectively. Next, an appropriate amount of toluene at $50-60^{\circ} \mathrm{C}$ was added and stirred until the asphalt and other soluble matter were completely dissolved in toluene. Then, the solution was poured into a sand core funnel with filter paper and washed with toluene to obtain CR powder until the filtrate was colorless.

After that, Part a, the rubber with filter paper was put into a measuring cylinder with a known volume of water $\left(V_{0}\right)$, and the increased volume $\left(V_{2}\right)$ was recorded. Simultaneously, the same operation on the pure CR was performed and the volume $\left(V_{1}\right)$ in the measuring cylinder was recorded. Part $b$, the washed $C R$ powder was placed in a vacuum drying oven with a temperature of $60 \pm 1^{\circ} \mathrm{C}$ and a vacuum of $93 \mathrm{kPa} \pm 1 \mathrm{kPa}$ to a constant weight, and the mass $\left(M_{1}\right)$ was recorded. Expansion ratio of TIM $(E)$, the TIM content $(K)$, and attenuation rate of TIM content $(Q)$ were calculated using equations (1)-(3), respectively:

$$
\begin{aligned}
E & =\frac{V_{2}-V_{0}}{V_{1}-V_{0}}, \\
K & =\frac{M_{1}}{c \times M_{0}} \times 100 \%, \\
Q & =\frac{K_{0}-K}{K_{0}} \times 100 \%,
\end{aligned}
$$

where $c$ is CR powder content (16\%) and $K_{0}$ is the content of TIM in pure CR powder in the experiment $(0.945 \%)$.

2.3.3. SEM. SEM has high resolution and can observe the microscopic morphology, structure, and distribution of modified asphalt $[21,22]$. The samples in the system were scanned under 20,000x magnification by Hitachi S- 4800 . 
TABLe 1: Physical properties of base asphalt.

\begin{tabular}{lccc}
\hline Properties & Softening point $\left({ }^{\circ} \mathrm{C}\right)$ & Penetration $\left(25^{\circ} \mathrm{C}, 0.1 \mathrm{~mm}\right)$ & Ductility $\left(15^{\circ} \mathrm{C}, \mathrm{cm}\right)$ \\
\hline Shell-70 & 47.5 & 72 & $>100$
\end{tabular}

TABle 2: Physical properties of crumb rubber.

\begin{tabular}{lcccc}
\hline Test methods & Relative density & Water content (\%) & Fiber content (\%) & Metal content (\%) \\
\hline Test value & 1.08 & 0.31 & 0.25 & 0.03 \\
\hline Screening test & & & & 0.15 \\
Sieve size (mm) & 0.6 & 0.3 & 2.4 & 0.075 \\
Trough rate $(\%)$ & 98.3 & 63.4 & 0.5 \\
\hline
\end{tabular}

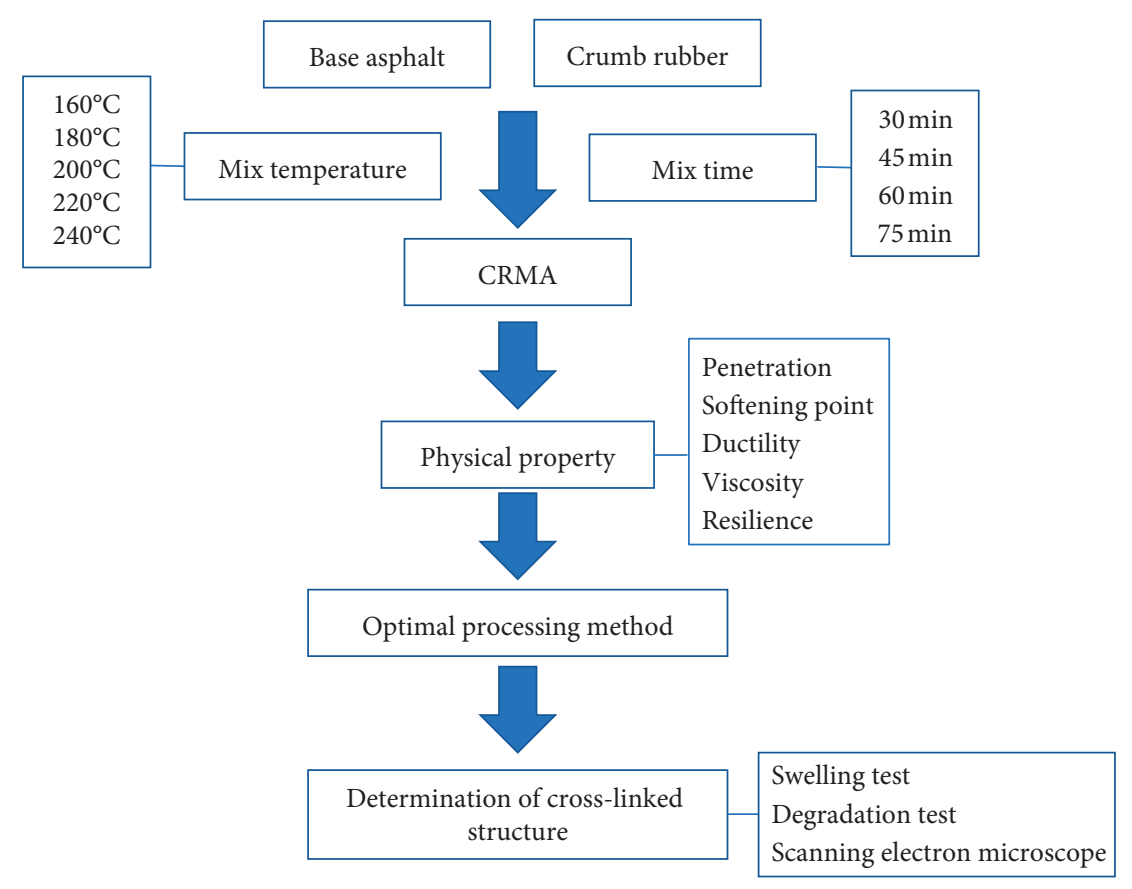

Figure 1: Experimental design.

\section{Results and Discussion}

3.1. The Influence of Processing Temperature on CRMA. Processing temperature is an important condition that affects the preparation process of CRMA. The higher the temperature, the better the reaction speed and degree of CR desulfurization and swelling, but too high temperature would easily lead to aging of the base asphalt and rapid CR degradation. Generally, when selecting the preparation temperature, the CR should be fully swelled and uniformly dispersed during the process. The control of specific temperature should be determined comprehensively according to the characteristics of base asphalt and rubber powder and their interaction. The influence of processing temperature on CRMA's performance is shown in Figure 2.

As the temperature increased, the penetration of CRMA basically showed an increasing trend (Figure 2(a)), because high temperature intensified the desulfurization and degradation of $\mathrm{CR}$, and the cross-linking reaction between $\mathrm{CR}$ and asphalt accelerated. Simultaneously, with the effective
CR particles decreasing, the soluble components in the asphalt increased, and the light components participating in the swelling reaction returned to the asphalt, resulting in more light components in the CRMA system at high temperatures than that at low temperatures, so the penetration degree increased with the increase of temperature.

From the data in Figure 2(b), as the processing temperature increased, the softening point decreased. The decreasing trend was not obvious between $180^{\circ} \mathrm{C}$ and $200^{\circ} \mathrm{C}$. Under this temperature range, the light components absorbed by the swelling reaction in CRMA, and part of CR underwent desulfurization and degradation reactions "freed" out soluble components; thus, they were in equilibrium. However, an inflection point occurred at $200^{\circ} \mathrm{C}$; that is, the softening point was greatly reduced. A qualitative change has occurred in CR interior at $200^{\circ} \mathrm{C}$. Desulfurization and degradation reactions began to replace swelling reactions and gradually dominate [23]. Therefore, when the preparation temperature was too high, the high-temperature stability of CRMA would decrease. 


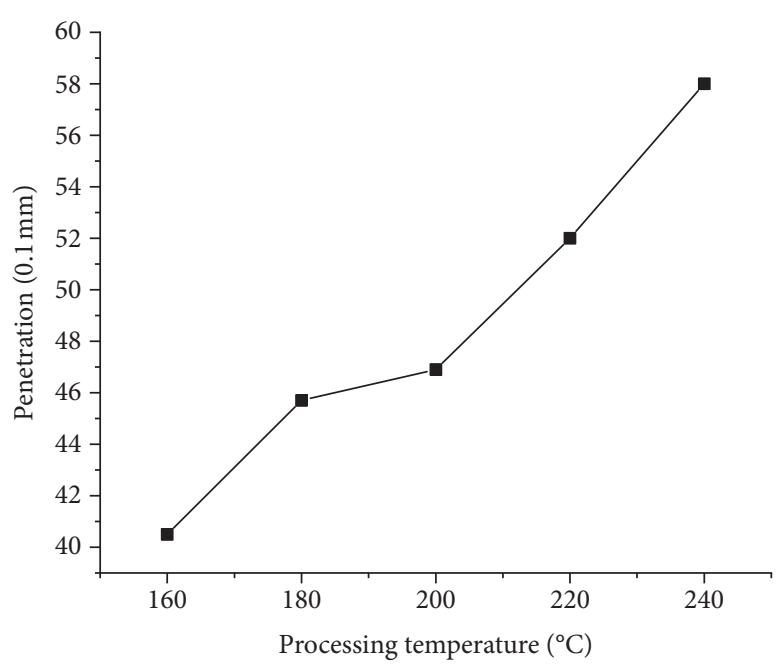

(a)

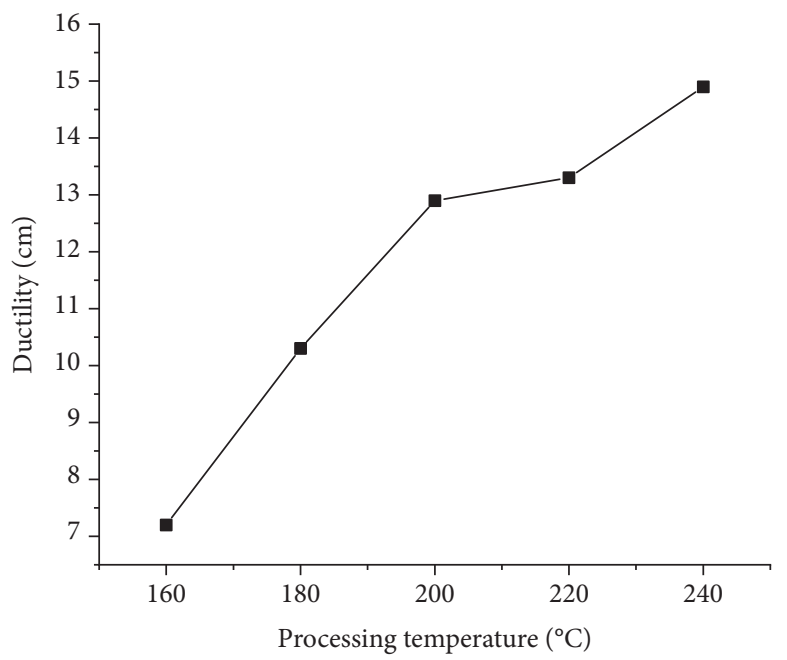

(c)

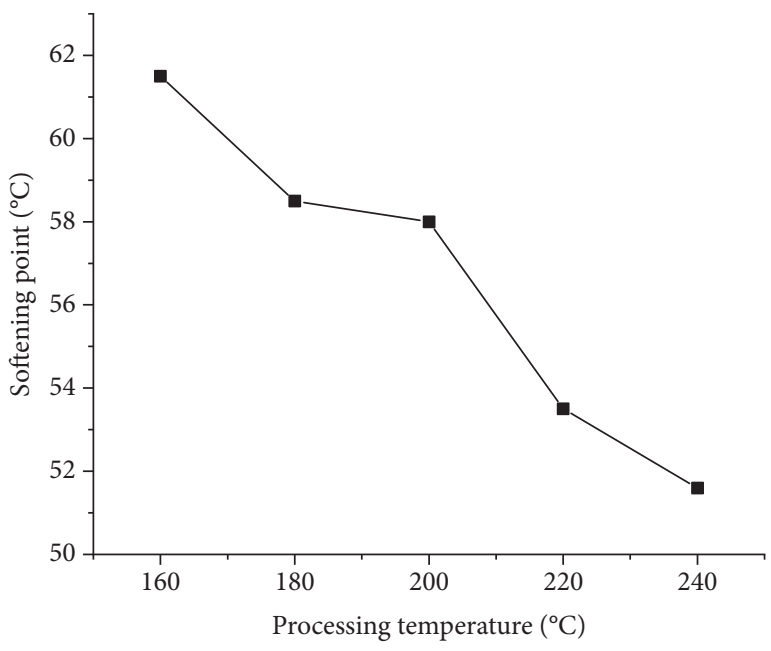

(b)

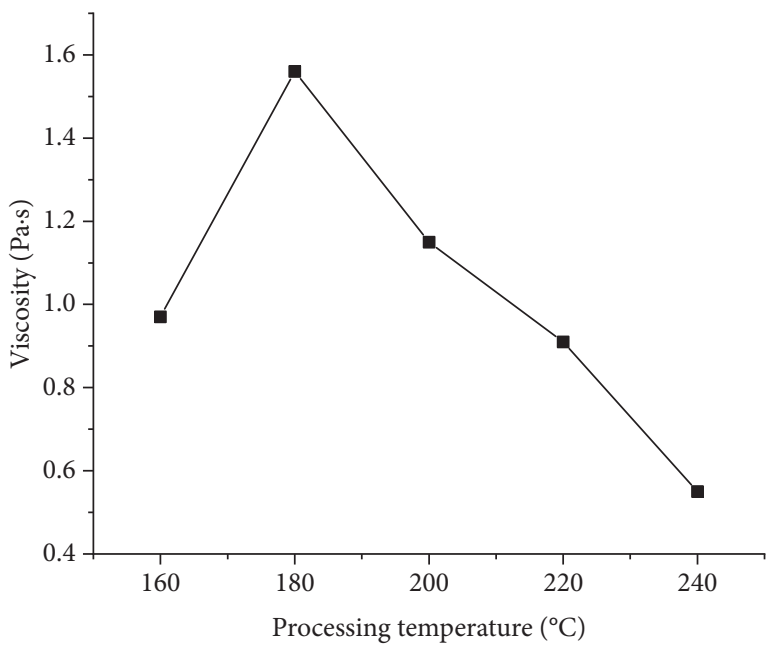

(d)

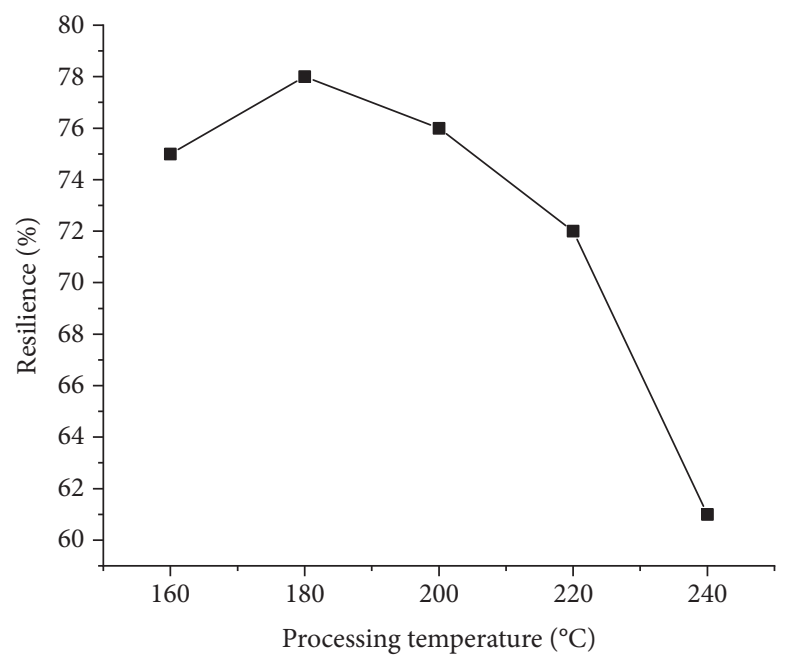

(e)

FIgURE 2: The influence of processing temperature on CRMA's performance. (a) Penetration. (b) Softening point. (c) Ductility. (d) Viscosity. (e) Resilience. 
The ductility of CRMA kept increasing within $200^{\circ} \mathrm{C}$, and basically tended to be stable after $200^{\circ} \mathrm{C}$ (Figure 2(c)). With the increase of temperature, the swelling rate of $\mathrm{CR}$ in asphalt gradually increased, and the volume of CR particles expanded rapidly. At the same time, the desulfurization and degradation effects increased the soluble composition of CR, which improved the low-temperature flexibility of CRMA. But when the temperature was higher than $200^{\circ} \mathrm{C}$, the asphalt began to age, and the light components were volatilized, while the degree of CR degradation also increased. Under the combined action, the trend of ductility was basically stable. After the temperature continued to rise, the rubber powder degraded seriously and lost the effect of improving the low-temperature performance of CRMA.

It can be seen from Figure 2(d) that the viscosity of CRMA first increased and then decreased. The swelling reaction rate of the rubber powder absorbing the light components in the asphalt increased, which enhanced the cross-linking between the $\mathrm{CR}$ particles, and finally increased the viscosity. After the temperature exceeded $200^{\circ} \mathrm{C}$, the desulfurization and degradation effects reduced the volume of the rubber powder. When the volume was small to a certain extent, the cross-linking between the CR particles weakened, thereby reducing the viscosity of CRMA.

The change trend of the elastic recovery of CRMA was shown to increase slowly and then decrease. At the beginning, the temperature rose, the swelling reaction rate increased, and CRMA formed a good micro-network structure, which enhanced the resistance to deformation. After the temperature reached $200^{\circ} \mathrm{C}$, the volatilization of light components intensified, and the micro-network structure was destroyed; thus, the elastic recovery decreased.

3.2. The Influence of Processing Time on CRMA. In the preparation process of CRMA, the processing temperature and processing time influence and restrict each other. Therefore, it is important to choose the right processing temperature and time. From Figure 3(a)-3(c), it can be seen that, with the extension of processing time, the penetration and ductility of CRMA basically showed an upward trend, while the softening point showed a downward trend. At the beginning of processing, CR mainly swelled in the asphalt, absorbed the light components, and formed a microscopic network structure with the asphalt. As the preparation time continued to extend, the swelling reaction reached the upper limit, and the desulfurization degradation of CR began to dominate. In the CRMA system, the effective rubber content decreased, so the penetration increased and the softening point decreased. The desulfurization and degradation reactions accelerated the generation of rubber's molecular chain segment.

The viscosity of CRMA showed a tendency to increase first and then decrease (Figure 3(d)), because the CR had poor swelling effect, small volume ratio, and discontinuous particles at the initial stage of preparation. After that, the continuous swelling reaction enhanced the mutual crosslinking between CR particles, resulting in an increase in viscosity. When the time was too long, the desulfurization and degradation would decrease the volume of the rubber particles and the effective rubber content. When the volume was small to a certain extent, the cross-linking between the $\mathrm{CR}$ particles would weaken, thus making the viscosity of CRMA decrease.

The resilience of CRMA gradually increased as a whole, and then its growth trend gradually slowed down. The volume of $\mathrm{CR}$ absorbed light components increased, and the elastic effect of rubber particles was enhanced, so that the resilience ability of CRMA was developed. After CR swelling reaction was sufficient, the elastic effect of CR particles was fully used, and the resilience of CRMA would slowly level off.

In general, when the processing temperature is too low or the mixing time is too short, the consistency of CRMA is relatively large, and the rubber powder cannot be fully dispersed in the asphalt, which is not conducive to the swelling reaction. Besides, too high temperature or too long time will cause excessive aging of asphalt and rapid degradation of rubber powder. In order to prevent excessive desulfurization and degradation of rubber powder, the final suitable processing technology determined according to the materials used is high-speed stirring at a temperature range of $180-190^{\circ} \mathrm{C}$ for $45-60 \mathrm{~min}$.

3.3. Degradation Analysis. During CRMA processing, due to the combined action of high-speed shear, high temperature, and time, rubber would swell, desulfurize, and degrade. The vulcanized rubber contained in the CR powder is insoluble and becomes soluble in toluene after desulfurization and degradation. The greater the attenuation rate ( $Q$ value) of toluene insoluble content, the more serious the desulfurization and degradation of CR powder. According to the test results in Figure 4, the $Q$ value went up with time. At the same time, the higher the processing temperature of CRMA, the greater the $Q$ value. It shows that the degree of desulfurization and degradation of rubber powder under hightemperature conditions was serious. With the extension of time, the degree of desulfurization and degradation continued to increase. The initial growth was relatively fast, but the later reaction gradually slowed down. Therefore, the reaction temperature should be strictly controlled in the CRMA processing. The low temperature and the insufficient swelling were not conducive to the desulfurization and degradation reaction. The insufficient sol content caused the improvement of the flexibility of the asphalt to be limited. This corresponded to the conventional physical properties of CRMA. However, too high temperature would cause excessive decomposition of rubber powder, so the processing temperature should not be too high, and the storage time under high temperature should not be too long.

3.4. Swelling Analysis. Crumb rubber would absorb the light components in asphalt and swell. It can be seen from Figure 5 that at low temperatures, as time increased, the expansion ratio gradually climbed and then tended to be flat. Due to the complex structure and large molecular volume of the saturated and aromatic components in asphalt, the content of light oil is limited, and it is also enveloped by gums and asphaltenes [24]. 


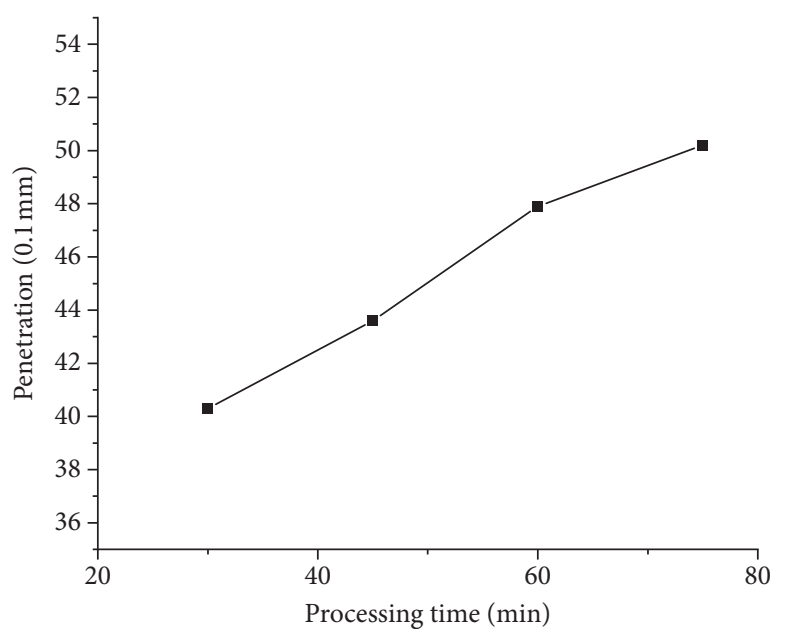

(a)

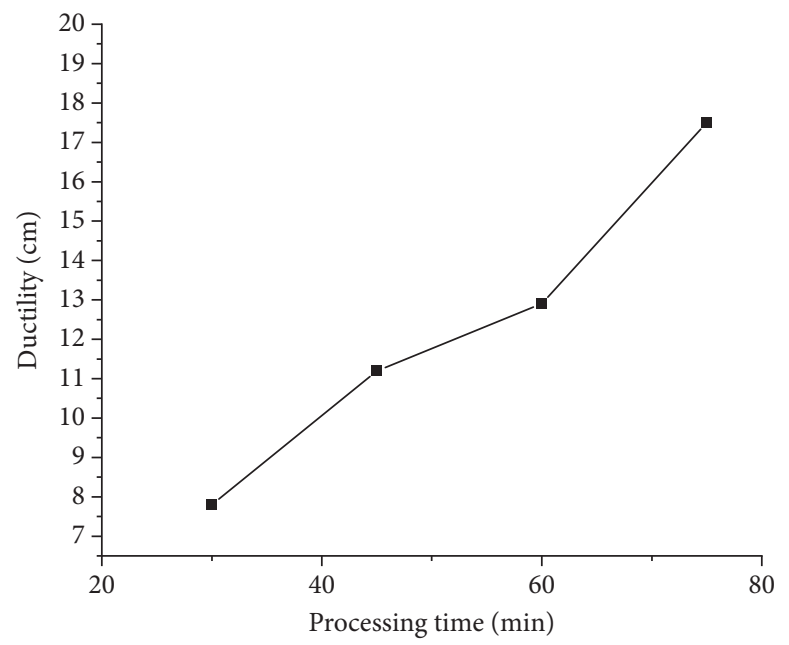

(c)

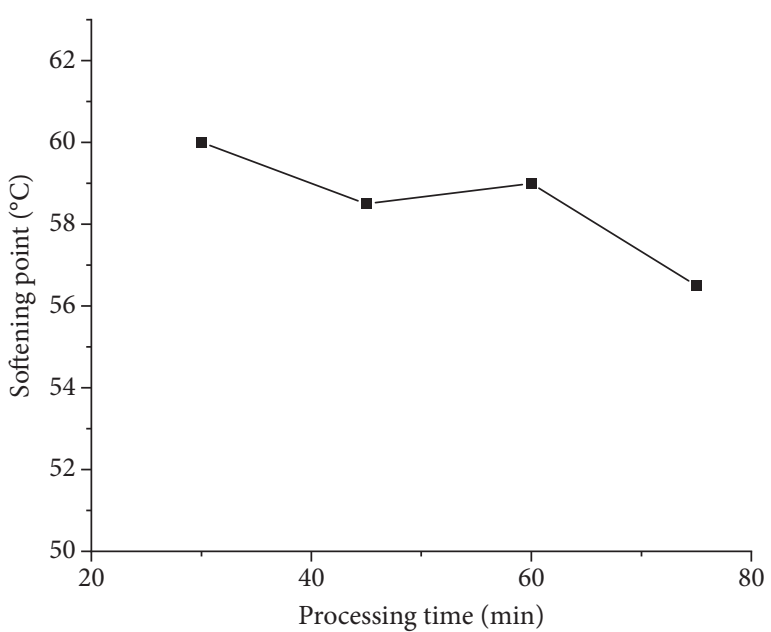

(b)

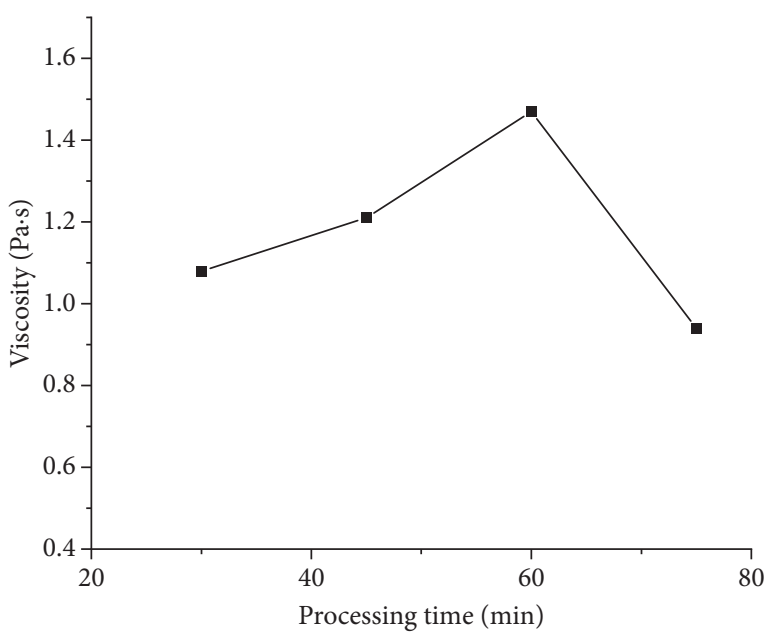

(d)

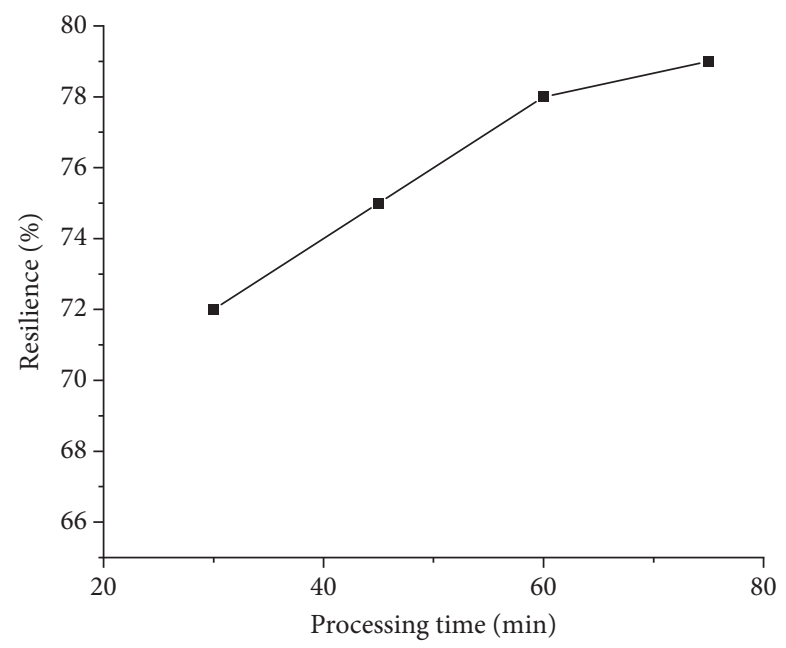

(e)

Figure 3: The influence of processing time on CRMA's performance. (a) Penetration. (b) Softening point. (c) Ductility. (d) Viscosity. (e) Resilience. 


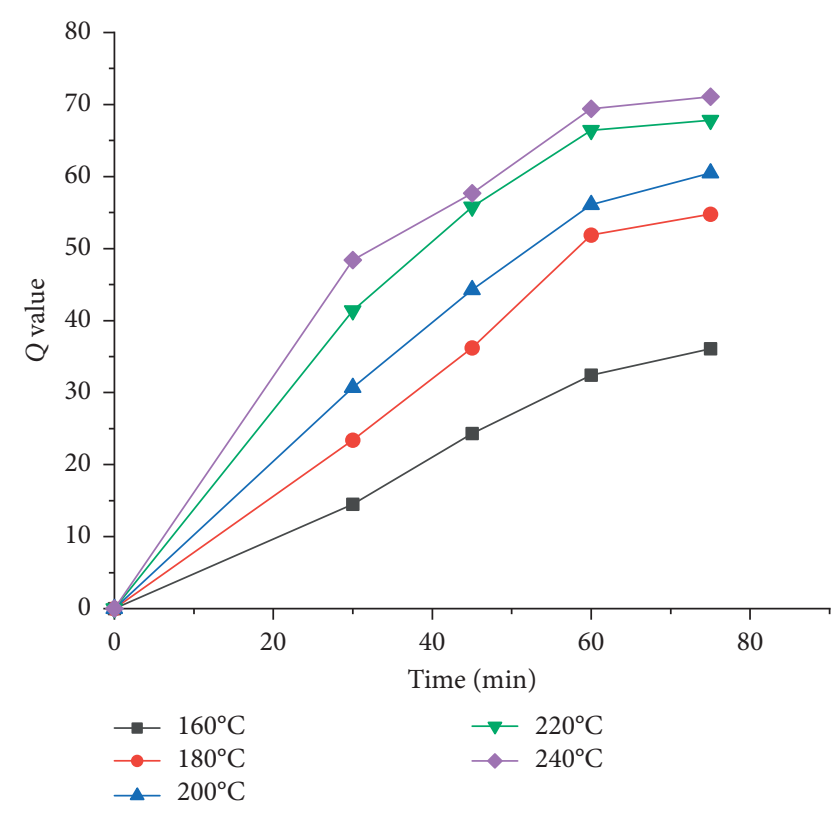

Figure 4: The changing law of toluene insoluble content attenuation rate.

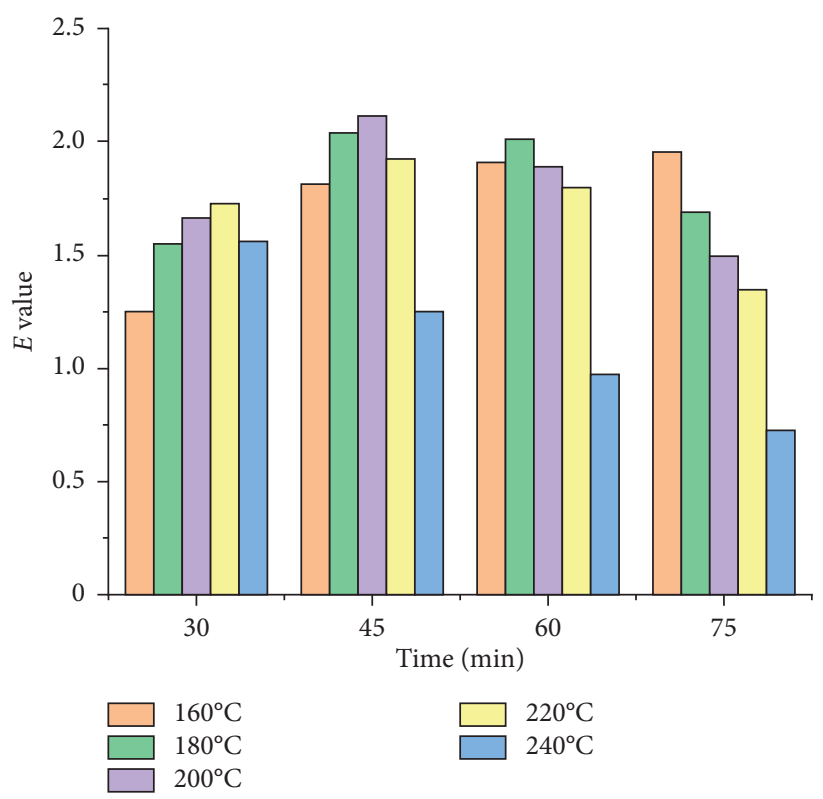

Figure 5: The expansion ratio of CRMA.

As the processing temperature increased, the speed of rubber swelling in asphalt rose. But if it is processed for a long time, the balance between swelling and desulfurization degradation would be broken. Evaluation by the volume of toluene insoluble matter shows that the expansion ratio of CRMA has decreased. When the temperature was too high, the speed of desulfurization and degradation was faster than that of swelling, which reduced the toluene insoluble matter, and the measured expansion ratio continued to decrease. Considering the large content of CR powder, the volume of CR powder after swelling in the asphalt was greatly increased, and because part of the light oil in the asphalt was absorbed by the CR powder, the total volume of the asphalt was reduced. The proportion of expanded rubber powder will account for $40 \%$ or more of the total volume of CRMA. The particles of rubber powder gradually change from the dispersed phase to the continuous phase, which leads to a large change in the properties of the asphalt.

3.5. Correlation Analysis of Swelling and Degradation. At this stage, the correlation between the swelling and degradation of CRMA at different temperature and time was analyzed, and the results are shown in Figure 6. At the same time, polynomial regression fitting on the data was performed, seen in Tables 3 and 4 for the fitting parameters. The closer the coefficient of determination $\left(R^{2}\right)$ value is to 1 , the better the fit of the regression line to the observed value; on the contrary, the smaller the value of $R^{2}$, the worse the fit of the regression line to the observed value. Due to the use of toluene insolubles to test the volume and content of vulcanizates, although there was slight error, the regularity was certain. For different processing times, when processing for $30 \mathrm{~min}$ and $75 \mathrm{~min}$, the correlation between the swelling and degradation of CRMA was better, and $R^{2}$ was above 0.90 . Since the swelling rate of CRMA was higher than that of degradation in a short time, and the opposite law appeared when the time is too long, the correlation is better. At $45 \mathrm{~min}$ and $60 \mathrm{~min}$, the balance of swelling and degradation of CRMA has been fluctuating; $R^{2}$ was only $0.64-0.72$. In addition, under different processing temperature conditions, the correlation of the swelling and degradation of CRMA at $160^{\circ} \mathrm{C}, 180^{\circ} \mathrm{C}, 200^{\circ} \mathrm{C}$, and $240^{\circ} \mathrm{C}$ was good; $R^{2}$ reached above 0.88. Simultaneously, it can be predicted from the fitting curve that, at $180^{\circ} \mathrm{C}$, the swelling rate is slightly faster than the degradation rate, while the degradation rate is faster than the degradation rate at $220^{\circ} \mathrm{C}$.

3.6. SEM. The morphology of the rubber powder particles was observed by SEM, and the results are shown in Figure 7, where (a) is the pure rubber powder particles, (b) is the observation of the rubber powder after washing with toluene, and (c) is observation that the rubber powder in CRMA was washed with toluene.

It can be seen from Figure 7 (a) that the surface of the pure rubber powder particles was uneven, with many wrinkles and a small amount of carbon black on the surface of the particles, which was approximately a network structure, resulting in easily adsorbing the light components in the asphalt. After being soaked and dissolved in toluene (Figure 7(b)), the CR powder particles were not completely dissolved, but no obvious changes were observed under the naked eye. SEM observations showed that the surface of CR particles had a small amount of change, surface wrinkles were more obvious, and carbon black particles increased. It indicates that some substances were dissolved under the action of toluene, making some carbon black particles exposed. After the CR powder was fully reacted in the asphalt (Figure 7(c)), the surface was more uneven and the exposed carbon black particles were more obvious. Under the action 


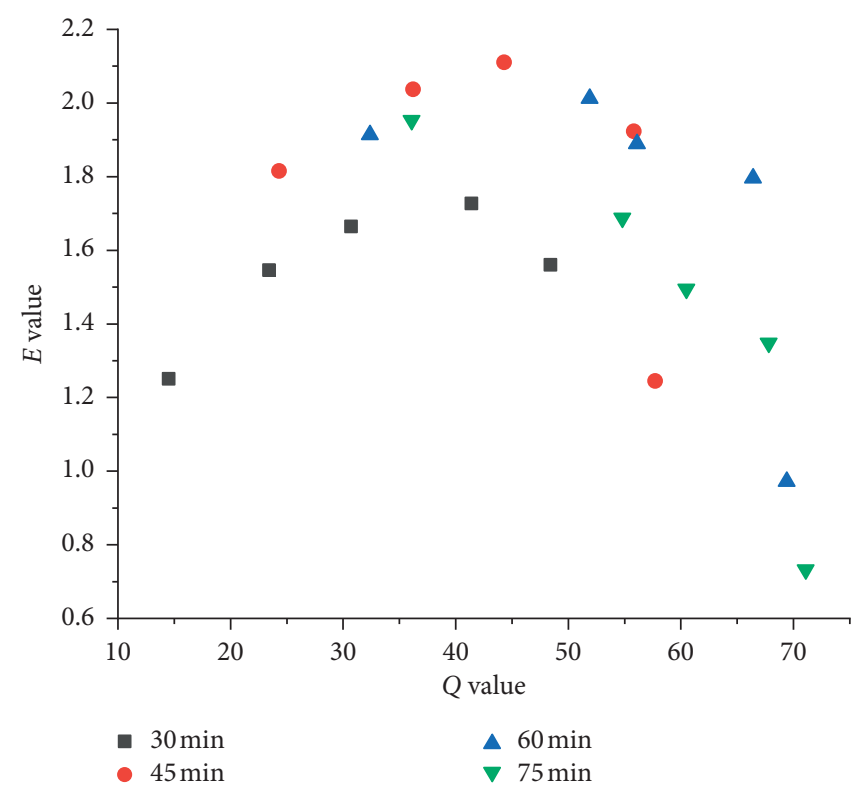

(a)

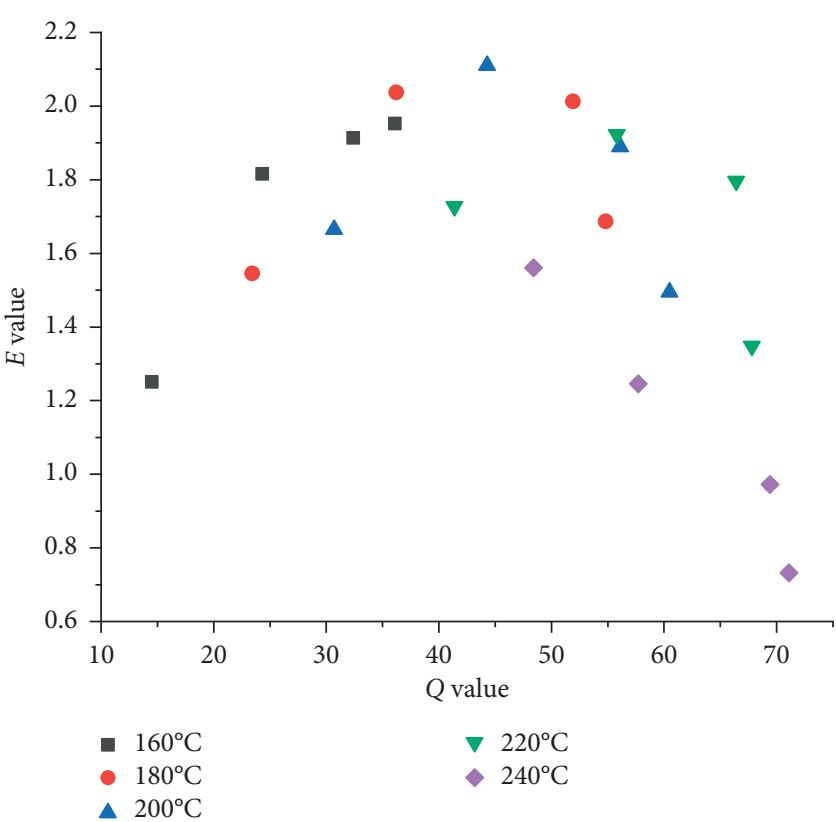

(b)

Figure 6: The relationship between Q value and E value. (a) Different time. (b) Different temperature.

TABLE 3: The fitting value of $Q$ value and $E$ value at different time.

\begin{tabular}{lcccc}
\hline Equation & & \multicolumn{2}{c}{$y_{2}=A+B_{1} * x+B_{2} * x^{2}$} & \\
Time & $30 \mathrm{~min}$ & $45 \mathrm{~min}$ & $60 \mathrm{~min}$ & $75 \mathrm{~min}$ \\
\hline $\mathrm{A}$ & $0.41709 \pm 0.10752$ & $-0.47394 \pm 1.76446$ & $-0.92246 \pm 2.3433$ & $-0.05313 \pm 1.94987$ \\
$\mathrm{~B}_{1}$ & $0.0713 \pm 0.00752$ & $0.13537 \pm 0.0905$ & $0.13604 \pm 0.09693$ & $0.09845 \pm 0.07659$ \\
$\mathrm{~B}_{2}$ & $-9.77807 E-4 \pm 1.17461 E-4$ & $-0.00175 \pm 0.00108$ & $-0.00151 \pm 9.49146 E-4$ & $-0.0012 \pm 7.16878 E-4$ \\
$R^{2}$ & 0.98564 & 0.64699 & 0.72062 & 0.90059 \\
\hline
\end{tabular}

TABle 4: The fitting value of $\mathrm{Q}$ value and $E$ value at different temperature.

\begin{tabular}{lccccc}
\hline Equation & & \multicolumn{3}{c}{$y_{1}=A+B_{1} * x+B_{2} * x^{2}$} \\
Temperature & $160^{\circ} \mathrm{C}$ & $180^{\circ} \mathrm{C}$ & $200^{\circ} \mathrm{C}$ & $220^{\circ} \mathrm{C}$ & $240^{\circ} \mathrm{C}$ \\
\hline $\mathrm{A}$ & $-0.24772 \pm 0.27814$ & $-1.0352 \pm 1.05358$ & $-2.78019 \pm 0.87009$ & $-3.73591 \pm 5.3311$ & $2.41475 \pm 4.52155$ \\
$\mathrm{~B}_{1}$ & $0.13284 \pm 0.02408$ & $0.15342 \pm 0.0584$ & $0.21996 \pm 0.04037$ & $0.21701 \pm 0.20182$ & $-0.00781 \pm 0.15374$ \\
$\mathrm{~B}_{2}$ & $-0.00201 \pm 4.75419 E-4$ & $-0.00187 \pm 7.37085 E-4$ & $-0.00246 \pm 4.42059 E-4$ & $-0.00206 \pm 0.00184$ & $-2.07267 E-4 \pm 0.00128$ \\
$R^{2}$ & 0.99331 & 0.88453 & 0.96963 & 0.62527 & 0.95613 \\
\hline
\end{tabular}

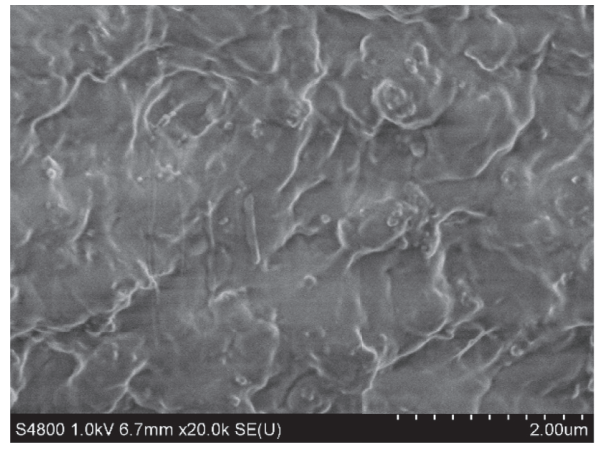

(a)

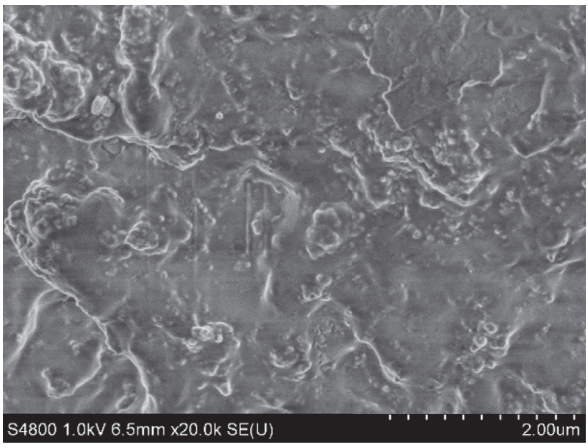

(b)

Figure 7: Continued. 


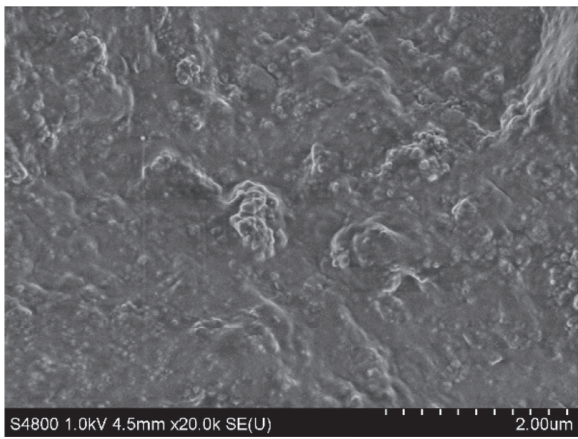

(c)

FIgURE 7: Observation results of rubber powder particle morphology. (a) Pure rubber. (b) Rubber after toluene rinse. (c) Rubber in the CRMA.

of high temperature and high-speed shear, part of the rubber particles desulfurized and degraded, showing off from the rubber powder, changing the original surface state [13].

\section{Conclusion}

(1) The processing temperature and time of CRMA should not be excessive; otherwise, they would lead to accelerated degradation of rubber and aging of asphalt and have a bad influence on the physical properties of CRMA. The final suitable processing technology was the temperature range of $180-190^{\circ} \mathrm{C}$ and the stirring time of 45-60 $\mathrm{min}$.

(2) With the increase of processing time and temperature, the attenuation rate of toluene insoluble matter went up, indicating that the rubber in CRMA was seriously degraded.

(3) For the swelling of rubber, at low temperature, the expansion rate increased as the time increased. But due to the effect of degradation at high temperature, the expansion rate decreased gradually with the increase of time.

(4) The expansion ratio of rubber and the attenuation rate of toluene insoluble matter showed a certain polynomial regression correlation at different processing temperature and time. Most of the correlation coefficients were above 0.88 .

(5) It can be seen from the SEM that excessive processing exposed the carbon black and accelerated the desulfurization and degradation of rubber in the CRMA.

\section{Data Availability}

Some of the data required to reproduce these findings cannot be shared at this time as the data also form part of an ongoing study.

\section{Conflicts of Interest}

The authors declare that they have no conflicts of interest regarding this work.

\section{Acknowledgments}

This research was supported by National Natural Science Foundation of China (51108374), Jiangxi Transportation Science and Technology Project (2016C0005), Key R\&D Program Funding Project of Shaanxi Provincial (2018SF380), and the Special Fund for Basic Scientific Research of Central Colleges, Chang'an University (300102310301).

\section{References}

[1] W. Huang and Y. Zhang, "The technical criteria frame of pavement used asphalt rubber in China," Journal of Central South Highway Engineering, vol. 32, no. 1, pp. 111-114, 2007.

[2] M. A. Mull, K. Stuart, and A. Yehia, "Fracture resistance characterization of chemically modified crumb rubber asphalt pavement," Journal of Materials Science, vol. 37, no. 3, pp. 557-566, 2002.

[3] Y. Yang, H. Yuan, and M. A. Tao, "Swelling principle and pavement performance of desulfurized rubber asphalt," Journal of Highway and Transportation Research and Development, vol. 29, no. 2, pp. 35-39, 2012.

[4] K. Baha Vural, M. Yilmaz, and A. Geçkil, "Evaluation of the low temperature and elastic properties of crumb rubber and SBS modified asphalt and mixtures," Journal of Materials in Civil Engineering, vol. 25, no. 2, pp. 257-265, 2013.

[5] Z. Ding and L. I. Pei-Long, "Research on influence factors and prediction model of viscosity of crumb rubber modified asphalt," Highway, no. 7, pp. 213-216, 2012.

[6] Y.-N. Cui, Y.-M. Xing, L. Wang et al., "Improvement mechanism of crumb rubber-modified asphalt," Journal of Building Materials, vol. 14, no. 5, pp. 634-638, 2014.

[7] H. Liu, G. Luo, X. Wang et al., "Effects of preparation process on performance of rubber modified asphalt," IOP Conf. Series: Materials Science and Engineering, vol. 87, Article ID 012008, 2015.

[8] T. Wang, R. Yang, A. Li et al., "Effects of sasobit and its adding process on the performance of rubber asphalt," Chemical Engineering Transactions, vol. 51, pp. 181-185, 2016.

[9] F. Ye, S. Yang, X. Wu et al., "Rheological property of highly degraded rubber modified asphalt," Journal of Building Materials, vol. 19, no. 5, pp. 945-949, 2016.

[10] Y. Liu, C. Liu, and Y. Zhang, "Reaction rules of crumb rubber modified asphalt," Journal of China University of Petroleum, vol. 41, no. 3, pp. 154-160, 2017. 
[11] A. Magdy and C. Samuel, "Mechanism of interaction of asphalt cement with crumb rubber modifier," Transportation Research Record: Journal of the Transportation Research Board, vol. 5, pp. 106-113, 1999.

[12] F. J. López-Moro, M. C. Moro, F. Hernández-Olivares, B. Witoszek-Schultz, and M. Alonso-Fernández, "Microscopic analysis of the interaction between crumb rubber and bitumen in asphalt mixtures using the dry process," Construction and Building Materials, vol. 48, pp. 691-699, 2013.

[13] P. Cong, P. Xun, M. Xing, and S. Chen, "Investigation of asphalt binder containing various crumb rubbers and asphalts," Construction and Building Materials, vol. 40, pp. 632-641, 2013.

[14] A. Ignacio and A. Khalid Hussain, "Diffusion kinetics of asphalt into waste tyre rubber (with discussion)," Association of Asphalt Paving Technologists, vol. 75, pp. 133-164, 2006.

[15] D. Dong, X. Huang, X. Li, and L. Zhang, "Swelling process of rubber in asphalt and its effect on the structure and properties of rubber and asphalt," Construction and Building Materials, vol. 29, pp. 316-322, 2012.

[16] P. Li, Z. Ding, and C. Chong, "Analysis of swelling and degradation characteristics of crumb rubber in hot asphalt," Journal of South China University of Technology (Natural Science Edition), vol. 44, no. 12, pp. 97-103, 2016.

[17] H. Wang, X. Liu, P. Apostolidis, S. Erkens, and T. Scarpas, "Numerical investigation of rubber swelling in bitumen," Construction and Building Materials, vol. 214, pp. 506-515, 2019.

[18] X. Ji, Y. Hou, and W. Han, "Establishment of swelling model and determination of swelling parameters between rubber and asphalt," China Synthetic Rubber Industry, vol. 33, no. 3, pp. 191-193, 2010.

[19] W. Zhang and K. Müllen, "Analyzing solid fossil-fuel pitches by a combination of Soxhlet extraction and Fourier transform ion cyclotron resonance mass spectrometry," Carbon, vol. 167, pp. 414-421, 2020.

[20] B. F. Bowers, B. Huang, and X. Shu, "New method for detecting asphalt contamination within fine aggregate medium through chemical testing," Journal of Materials in Civil Engineering, vol. 25, no. 2, pp. 252-256, 2013.

[21] H. Hong, H. Zhang, and L. Huang, "Study progress of characterization of asphalt materials by nuclear magnetic resonance, thermal analysis and scanning electron microscopy," Journal of Highway and Transportation Research and Development, vol. 36, no. 12, pp. 15-28, 2019.

[22] M. Mithil, R. Ahmed, A. Ahmed et al., "SEM and ESEM techniques used for analysis of asphalt binder and mixture: a state of the art review," Construction and Building Materials, vol. 186, pp. 313-329, 2018.

[23] L. He, T. Ling, Y. Ma et al., "Research on asphalt rubber aging performance and characteristics," Journal of Building Materials, vol. 18, no. 4, pp. 565-571, 2015.

[24] R. Dong, J. Yang, and M. Zhao, Research on New Component Analysis Method for Pyrolytic Crumb Rubber Modified Asphalt, Transportation Research Congress, Hangzhou, China, 2017. 